\title{
Direct quantitative judgments of sums and a two-stage model for psychophysical judgments'
}

\author{
DWIGHT W. CURTIS ${ }^{2}$ AND BEN E. FOX \\ CALIFORNIA STATE COLLEGE AT FULLERTON
}

\begin{abstract}
Judged magnitudes of differences between stimuli have previously been shown to support a two-stage interpretation of magnitude estimation, in which input transformations and output transformations are each describable as power functions. In an effort to provide support for the model independent of the difference estimation procedure, the present investigation employed two additional judgment tasks. We obtained magnitude judgments and category judgments of the combined magnitudes (sums) of paired weights from two groups of $S s$. Values of the inferred input exponent $k$ calculated from the two sets of data were very similar and were also remarkably similar to the exponent previously calculated from magnitude estimations of differences between weights. The output exponent calculated from magnitude judgments of sums described a concave upward function; however, the similar function describing category judgments was essentially linear. These results show that the inferred input exponent is not the result of the difference estimation task, and in addition provides support for the contention that the interval scale may be a less biased sensory measure than the magnitude scale. The introduction of an additive constant to the model improved its fit to the data but the rule by which it was introduced made very little difference.
\end{abstract}

Direct judgments of stimulus magnitudes have been extensively employed in sensory scaling. It has been argued that the measures so constructed represent ratio scales which directly reflect transducer processes (Stevens, 1961). Alternative scaling procedures, employing judgments of intervals between stimuli, have fallen into relative disrepute because scales constructed by these procedures are nonlinear with the magnitude estimation scales. Several recent writers (e.g., Attneave, 1962; Ekman, 1964; Torgerson, 1961; Treisman, 1964), however, have questioned the directness of magnitude estimations, suggesting that the power law,

$$
\mathrm{J}=\mathrm{a} \phi^{\mathrm{n}},
$$

describing the relationship between numerical judgments and physical stimulus values confounds a sensory or input function with an output function having to do with the way in which Ss assign numbers to perceived magnitudes.

The present paper considers magnitude judgments and category judgments from the standpoint of a two-stage model in which input and output are each described by power transformations. The appropriate function relating judgments to stimulus values, according to this model, would be of the form

$$
\mathrm{J}=\mathrm{a} \phi^{\mathrm{km}}
$$

where $\mathrm{k}$ and $\mathrm{m}$ describe transformations taking place in input and output, respectively.

From the data of the usual magnitude estimation experiment, there is no way to determine the unique values of $\mathrm{k}$ and $\mathrm{m}$; however, there are other judgment operations which make possible their separation. For instance, we may obtain magnitude estimations of the intervals between paired stimuli. If $\mathrm{Ss}$ judge relative differences between perceived magnitudes in the same way that they judge relative apparent magnitudes, a sensory transformation should presumably operate upon individual stimulus values, but any transformation occurring during output should operate upon perceived differences. Consequently, the relationship between numerical judgments of differences and stimulus values would have the form,

$$
\mathrm{J}_{\mathrm{ji}}=\mathrm{a}\left(\phi_{\mathrm{j}}^{\mathrm{k}}-\phi_{\mathrm{i}}^{\mathrm{k}}\right)^{\mathrm{m}},
$$

where $J_{j i}$ represents a numerical judgment of the difference between Stimulus $i$ and Stimulus $j$, and $k$ and $m$ are the input exponent and the output exponent, respectively. The exponents $\mathrm{k}$ and $\mathrm{m}$ in $\mathrm{Eq}$. (3) are mathematically independent and can be estimated by standard curve-fitting procedures. Since, according to Eq. (1) and Eq. (2), $n=k m$, we may estimate a value $\mathrm{n}$ from the difference estimation exponents which should predict the value of $n$ obtained by fitting Eq. (1) to magnitude estimations of stimuli.

In a series of experiments (Curtis, 1967; Curtis, Attneave, \& Harrington, 1968; and also several unpublished studies), we have calculated the exponents $k$ and $m$ from magnitude estimations of differences between stimuli from several sensory modalities. The two exponents in combination predict quite well magnitude estimations of stimuli on the same sensory continua. Values of the input exponent $k$ for a given continuum were significantly less variable than the output exponent $\mathrm{m}$, both across Ss and across experiments. The typical value of $\mathrm{k}$ was smaller than the exponent described by Stevens for the same modalities, and the output exponents were typically greater than 1.0 .

In other experiments Ss made category judgments of differences between stimuli, as well as category judgments of the same stimuli. The exponents calculated from difference judgments predicted well category judgments of the same continua, but the output exponents calculated from category judgments described essentially linear relationships between perceived differences and scale values. This suggests that the category scale may more accurately reflect an underlying sensory process.

The present experiment was conducted to determine whether the results of these previous investigations could be obtained from judgment tasks other than the difference estimation task (e.g., Ss may have adopted something like Torgerson's (1961) "difference strategy"). Two groups of Ss estimated the combined magnitudes (sums) of stimuli according to magnitude instructions, or according to category judgment instructions. An extension of our model to these "summation" judgments would have the form,

$$
\mathrm{J}_{\mathrm{ij}}=\mathrm{a}\left(\phi_{\mathrm{i}}^{\mathrm{k}}+\phi_{\mathrm{j}}^{\mathrm{k}}\right)^{\mathrm{m}}
$$

Previous research has shown that Eqs. (1) and (3) fit the data better where they are modified to incorporate additive constants. The meaning of the constant and the form of the function in which it is implicated has been the subject of some debate (see Fagot, 1966, for a discussion of this issue). In earlier studies, we have employed the assumption that the correction largely reflects a constant error in S's response. This assumption led to modification of Eq. (3),

$$
\mathrm{J}_{\mathrm{ji}}=\mathrm{a}\left(\phi_{\mathrm{j}}^{\mathrm{k}}-\phi_{\mathrm{i}}^{\mathrm{k}}\right)^{\mathrm{m}}+\mathrm{b}
$$


where $b$ represents a correction on the response scale. Generalized to the summation judgment, this interpretation would imply the equation

$$
\mathrm{J}_{\mathrm{ij}}=a\left(\phi_{\mathrm{i}}^{\mathrm{k}}+\phi_{\mathrm{j}}^{\mathrm{k}}\right)^{\mathrm{m}}+\mathrm{b} .
$$

More frequently, however, the constant is treated as a threshold parameter, introducing it to the power function either (1) by translation on the stimulus axis (e.g., Stevens, 1961), or (2) by translation on the response axis (e.g., Fagot \& Stewart, 1968). The summation function consistent with (1) would be

$$
\mathrm{J}_{\mathrm{ij}}=\mathrm{a}\left[\left(\phi_{\mathrm{j}}-\mathrm{c}\right)^{\mathrm{k}}+\left(\phi_{\mathrm{j}}-\mathrm{c}\right)^{\mathrm{k}}\right]^{\mathrm{m}}
$$

where the constant $\mathrm{c}$ is interpreted as the effective threshold. Alternative (2) implies the equation ${ }^{3}$

$$
\mathbf{J}_{\mathrm{ij}}=\mathrm{a}\left(\phi_{\mathrm{i}}^{\mathrm{k}}+\phi_{\mathrm{j}}^{\mathbf{k}}-2 \mathrm{~d}\right)^{\mathrm{m}},
$$

where $d$ is a function of the threshold, $d=c^{k}$.

Since the constant has been subject to conflicting interpretations and these interpretations might well be expected to influence the forms of the input function and output function of the two-stage model, the empirical implications of each of the above assumptions were considered in the present investigation.

\section{Subjects}

\section{METHOD}

Two independent groups of Ss participated, 11 in the magnitude estimation experiment and 10 in the category judgment experiment. All Ss were volunteer students or wives of students. None of the Ss had had previous experience in scaling experiments.

\section{Apparatus}

Excepting that a new series of weights was prepared, the apparatus employed in the present experiment was essentially similar to that used by Curtis, Attneave, and Harrington (1968). It consisted of a plywood board, 18 in. x 24 in., through which four nylon lines extended through 1/8-in. holes. Plastic rings were attached to the upper end of the lines, which $\mathrm{S}$ grasped in lifting the weights. Wire hooks were attached to the lower extremes of the lines from which the weights were suspended. The arrangement facilitated the interchange of weights and removed the weights from the view of the $S$.

Eight weights were selected: $10.1,25.6,46.3,70.0,100.9$, $135.0,172.8$, and $217.7 \mathrm{~g}$. These were chosen to approximate an equal spacing on a power of .6 scale. Small errors were made in the construction of the stimulus series which were not discovered until the experiments were under way. Since the largest of these was approximately a gram $(70.0 \mathrm{~g}$ and $217.7 \mathrm{~g}$ ), it was decided to make no changes. The eight weights were paired to form 28 stimuli, consisting of all combinations of two weights.

\section{Procedure}

Category judgments. The category judgment experiment was conducted first. $S$ was instructed to lift two weights, suspended from the nylon lines, by successively lifting the two plastic rings with his preferred hand. He was permitted to lift the weights as many times as he required to arrive at his judgment; but he was instructed to lift each successively beginning with the weight on his left so exposure to the two weights would be approximately equal. Each weight pair was presented five times. Stimulus presentations were randomized within series of 28 , and with respect to the position of the heavier weight. The detailed instructions were as follows:
"In this experiment I would like you to estimate the combined weights of two stimuli when they are given to you at the same time. I will place two weights at the end of these strings so that you can lift them by pulling on these tabs with your hand (demonstrates). Use your favored hand (right or left). You may lift the weights as many times as you need to make a judgment, but always begin with the right weight and end with the left weight, so that each weight is lifted an equal number of times.

"Your judgment will be a rating of how heavy the two weights are in combination, on a scale of 1 to 11 . If the combined weights seem quite small, give me a small number, like 1 or 2 . If the combined weights seem quite heavy, give me a large number, like 10 or 11 . In assigning numbers, equal differences between numbers should represent about equal differences between the combined weights of pairs of weights. Do you understand?

"The heaviest and the lightest of the combinations will be presented first, so you can orient yourself. Call the lightest pair 1 and the heaviest pair 11 .

"Try to make your judgments independent, so that each judgment represents how heavy the pair feels at the present time. If a particular pair resembles a pair you have previously lifted, don't try to remember the number you assigned to the previous pair. Tell me a number that seems appropriate now.

"Any questions?

"All right, here is the lightest pair (demonstrates), and the heaviest pair (demonstrates)."

Magnitude estimation. In the magnitude estimation experiment, two weights were employed as a standard pair and were presented on every trial together with two weights which constituted the comparison stimulus. The two standard weights were $172.8 \mathrm{~g}$ and $217.7 \mathrm{~g}$. S was instructed to call the sum of their apparent magnitudes 25 . The choice of this pair, the heaviest, as a standard resulted from pilot observations of Ss, given a standard intermediate in the series, who reported that when the ratio of the comparison stimulus to the standard stimulus was greater than unity there was a temptation to assign numbers to the separate weights of the pair rather than to the magnitude of the pair. Analysis of these $S s^{3}$ data suggested that something of the sort might be occurring; observations where the ratio of the comparison pair to the standard pair was smaller than 1.0 appeared to be described by a function rather different from the function describing observations where the ratio was greater than 1.0. Ss were questioned after they had completed the experiment, and it appeared that by restricting the stimulus set to ratios smaller than unity this tendency was largely eliminated.

Due to an administrative error, five of the Ss were presented with each comparison pair six times and the remaining $S$ s were presented with each pair five times. Stimulus presentations were randomized within series and with respect to the position of the heavier stimulus.

The instructions to the Ss were as follows:

"In this experiment, I would like you to estimate the combined weight of pairs of weights. I will place two weights on the end of these strings so that you can lift them by pulling on these tabs with your hand (demonstrate). Use your favored hand (right or left). You may lift the weight as many times as you need to make a judgment, but always begin with the right weight and end with the left weight, so that each weight is lifted an equal number of times.

"In making your judgment, I would like you to give me a number which represents the combined weights of the two you have lifted. You will decide what number is appropriate by comparing the two weights with a standard pair. By pulling on these two tabs you can lift the standard weights (demonstrate). We will call their combined weights 25 . Lift the standard and then the comparison weights and give me a number which represents how heavy the comparison weights 
are, relative to the standard. If the combined comparison weights seem to be about half as heavy as the combined standard weights, give me a number about half as large, such as 12 or 13 . If the combined comparison weights appear to be about $1 / 10$ as large, give me a number like 2 or 3 , etc. The comparison total will always be smaller than the standard.

"Remember, you use a single number to represent the combined weights; do not try to assign values to the individual weights, but only to your total impression of heaviness from the two weights.

"Try to make your judgments independent, so that each judgment represents how heavy the comparison pair seems, relative to the standard pair. If a particular comparison pair resembles a pair you have previously lifted, don't try to remember the number that you assigned to the previous pair; let the number that you give me represent how heavy the pair seems right now.

"Any questions?"

\section{Magnitude Estimations}

\section{RESULTS AND DISCUSSION}

The magnitude estimations of the 27 stimulus pairs were averaged by first calculating the geometric mean of each S's response, and then determining the geometric mean of these averages. By using this sequential procedure each S's responses were weighted equally, even though some Ss provided a greater number of responses than did others.

Functions were fitted to the data by an iterative, least-squares procedure, employing an IBM 1620 computer. This method determines the values of the parameters $A_{1}, \cdots, A_{r}$, by minimizing the function

$$
Q=\sum_{i=1}^{28} w_{i}\left[J_{i j}-f\left(\phi_{i 1}, \phi_{i 2} ; A_{1}, \cdots, A_{r}\right)\right]^{2} .
$$

In the above equation, $\mathrm{J}_{\mathrm{ij}}$ is the dependent variable, the geometric mean of judgments of the sum of the two independent variables with values $\phi_{\mathrm{i} 1}$ and $\phi_{\mathrm{i} 2}$, and fepresents the theoretical function, the model implied by the specific assumptions concerning the presence or absence of an additive constant. The $\mathrm{w}_{\mathrm{i}}$ were weights assigned to observations. In the present analyses all $\mathrm{w}_{\mathrm{i}}$ equaled 1.0 ; all observations were equally weighted. That is, the line was fitted to the observations rather than to their logarithms. The decision to minimize the squared deviations of observations rather than the squared deviations of their relative magnitudes [as in the case of the use of logarithms, which in the present instance would make $\left.w_{i}=(1 / Y)^{2}\right]$ was based upon tests of the homogeneity of variance indicating that homoscedasticy was approximated by the distribution of the observations themselves $\left(\chi^{2}=21.74\right.$, df $\left.=26\right)$, but not by the distribution of logarithms $\left(\chi^{2}=86.05, p<.001\right)$. The finding of homogeneity of the variance of magnitude estimations, a rather unusual finding, is probably a consequence of employing the largest stimulus pair as standard. This strategy tended to reduce the variability of responses to stimuli adjacent to the standard, thereby inhibiting the usual tendency for variability to increase as the stimulus magnitude increases.

In analyzing the data we asked two questions. First, does a two-stage power model provide a satisfactory description of magnitude estimations of sums which is also consistent with the results of a similar analysis of magnitude estimations of differences? Second, do the several possible assumptions about the necessity for and meaning of the additive constant have empirically discriminable consequences? Four functions were fitted to the data: the three-parameter function, Eq. (4), and the three four-parameter functions, Eqs. (5), (6), and (7). Table 1 lists the estimates of the parameters with their associated S.E.s for the four equations.
Table 1

Parameter Values for the Alternative Forms of the Two-stage Model Parameter Values

\begin{tabular}{lcccc} 
Equation & $\mathrm{k}$ & $\mathrm{m}$ & $\mathrm{c}$ & $\mathrm{b}$ \\
\hline $\begin{array}{l}(4) \\
\text { No } \text { Constant }\end{array}$ & $.684 \pm .057$ & $1.403 \pm .102$ & & \\
$\begin{array}{c}(5) \\
\text { R-model } \\
(6)\end{array}$ & $.657 \pm .044$ & $1.202 \pm .075$ & & \\
RT-model & $.658 \pm .066$ & $1.196 \pm .081$ & $-6.337 \pm 2.413$ & \\
$\begin{array}{c}(7) \\
\text { ST-model }\end{array}$ & $.716 \pm .061$ & $1.215 \pm .108$ & $7.333 \pm 2.751$ & \\
\hline
\end{tabular}

Examine first the parameters $\mathrm{k}$ and $\mathrm{m}$ of $\mathrm{Eq}$. (5). This is the summation judgment generalization of the function (R-model) fitted to interval estimations of weights by Curtis, Attneave, and Harrington (1968). According to this analysis magnitude judgments of sums are best fitted by the function

$$
\mathrm{J}_{\mathrm{ij}}=.181\left(\phi_{\mathrm{i}} .657+\phi_{\mathrm{j}} .657\right)^{1.202}-2.408 \text {. }
$$

The exponent $\mathrm{k}$ of .657 is almost identical to the value .645 resulting from the analysis of difference judgments. This remarkable convergence of two independent estimates of an inferred sensory input parameter complements the earlier finding that the model also predicted the relationship between magnitude estimations of weights and magnitude estimations of weight differences with a comparable accuracy. On the other hand, the exponent $\mathrm{m}$ of 1.202 is consistent with a slightly, but reliably, curvilinear output function; $m$ differs from unity by slightly more than twice its S.E.

Excepting for Eq. (4), the three-parameter function, it is evident in Table 1 that the form of the model has rather little effect upon the values of $\mathrm{k}$ and $\mathrm{m}$. Eq. (4) where the function is forced through the origin requires a larger exponent $m$ than the other models; however, its exponent $\mathrm{k}$ differs only slightly from the others. The three four-parameter functions are essentially similar in their values of $\mathrm{m}$, although $\mathrm{Eq}$. (7), where the threshold parameter is translated on the stimulus axis (TS-Model), exhibits a slightly larger value of $\mathrm{k}(.716)$, as would be expected.

An analysis of variance of regression was performed to test the goodness-of-fit of each function to the data. The F statistics for deviations from regression are summarized in Table 2. None of the four $F$ ratios are significant; all four functions may be regarded as consistent with the data. It is not surprising that the largest of these statistics is obtained by the three-parameter function, Eq. (4). The geometric means of the magnitude estimations are shown as a function of values on Eq. (4) in Fig. 1B. This plot may be compared with Fig. 1 A where the same data are shown as a function of values on Eq. (5). The larger deviation $F$ ratio in the former case originates in the poorer fit at small stimulus values where Fig. 1B is slightly curvilinear. ${ }^{4}$ On the other hand, the similarity of the parameters and the deviation $F$ ratios

Table 2

F Values ${ }^{\mathrm{a}}$ for Analysis of Variance of Regression

\begin{tabular}{cc}
\hline Equation & F-Deviations \\
\hline $\begin{array}{c}(4) \\
\text { No constant } \\
(5)\end{array}$ & 1.45 \\
R-model & 1.28 \\
$(6)$ & 1.28 \\
RT-model & $(7)$ \\
ST-model & 1.30 \\
\hline
\end{tabular}

$a_{d f}=26 \& 280$ 


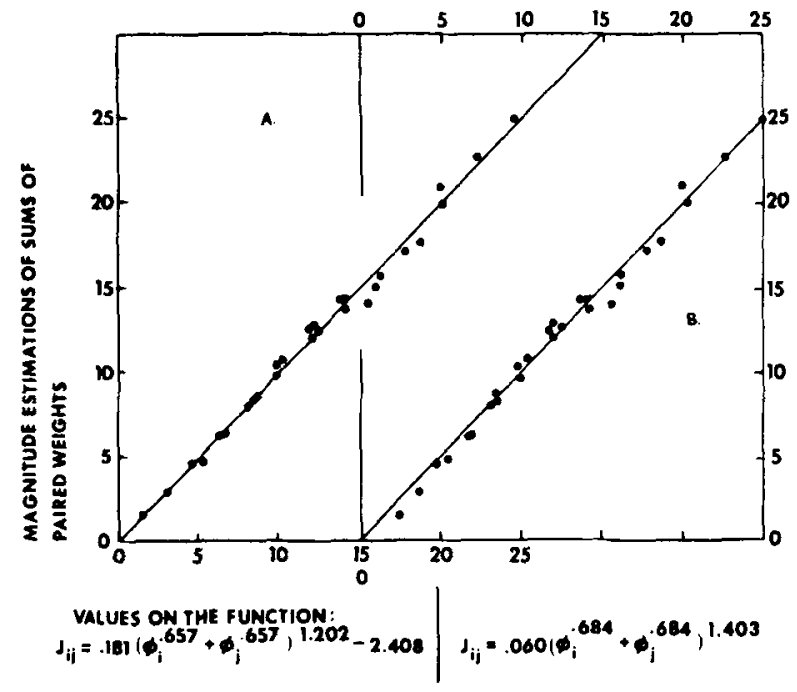

Fig. 1. Judgments of the combined magnitude of pairs of weights as a function of values predicted by (A) the four-parameter R-Model, Eq. (5) (B) the three-parameter model, Eq. (4).

associated with the remaining functions suggests that it doesn't much matter how the constant is added, as long as it is present. The particular form of the model may have greater significance, however, where stimuli more nearly approach absolute threshold level. Neither should the possibility be overlooked that an effective threshold may be confounded with constant errors on the response. Fagot and Stewart (1968) had Ss judge the magnitudes of stimuli close to the threshold for brightness. Additive constants estimated simultaneously with the exponent of the power function by a method similar to ours were much larger than either the measured absolute threshold or an effective threshold, measured in the presence of the standard. The power function provided a significantly better fit when a threshold parameter was introduced on the response axis than when it was translated on the stimulus axis. The response translated threshold constant, however, would drop out where magnitude estimations were of differences between stimuli; apparent magnitudes of differences should be independent of the

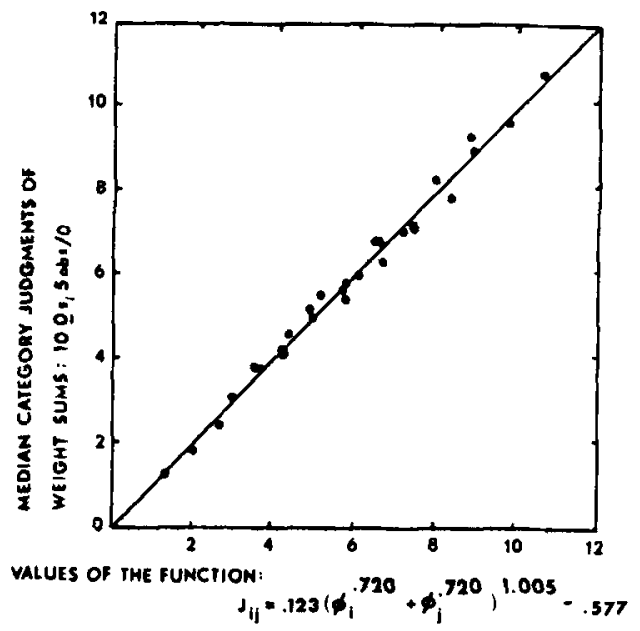

Fig. 2. Category judgments of sums of weights as a function of values predicted by the model (Eq. 5). threshold, as in Eq. (3), above. Curtis, Attneave, and Harrington (1968) obtained a better fit to magnitude judgments of differences between lifted weights when such a constant was considered, and for some Ss the magnitude of the correction was quite substantial.

\section{Category Judgments}

Equation (5) was the only function fitted to the category judgment data since, theoretically, these judgments are values on an interval scale, at best. Although any threshold assumptions applicable to magnitude estimations should also apply to category judgments, Eq. (5) was used exclusively because: (1) any consideration of a threshold would require a five-parameter function, and (2) Eq. (5) provides at least as good a fit to the magnitude estimations as any other four-parameter model.

A least squares analysis of the pooled category judgment data yielded the function

$$
\mathrm{J}_{\mathrm{ij}}=.123\left(\phi_{\mathrm{i}}^{.720}+\phi_{\mathrm{j}}{ }^{720}\right)^{1.005}-.578 .
$$

The fit of the function to the data is excellent, as is shown in Fig. 2. An analysis of variance of the deviations from regression was not significant: $F=1.34, \mathrm{df}=26,252$.

The value of the exponent $\mathrm{k}, .720 \pm .064$, is very similar to the value of this exponent calculated from magnitude estimations, differing by .063 when the parameters of the same equation (Eq. 5) are considered. Both of these estimates are quite similar to the exponent with the value .645 obtained by Curtis, Attneave, and Harrington (1968) from magnitude estimations of differences between weights. This degree of convergence is quite remarkable, particularly when it is considered that independent groups were tested in each task, the difference estimation experiment employed a different stimulus spacing and no standard, and, finally, that previous investigations employing magnitude judgments of lifted weights have yielded highly variable results. On the other hand, the output exponent $\mathrm{m}$ calculated from category judgments of sums describes an essentially linear output relationship which contrasts with the slight, but reliable, curvilinearity described by the output exponent of approximately 1.2 obtained from magnitude judgments.

The present data support the interpretation that the difference between magnitude estimations and category judgments is principally on $\mathrm{m}$. In this, it is consistent with our previous findings. In addition, the nonlinearity of the output relationship in the case of magnitude estimations suggests the interpretation that numerical judgments of the relative magnitudes of stimuli introduce biases which are relatively less evident in category judgments.

\section{REFERENCES}

ATTNEAVE, F. Perception and related areas. In S. Koch (Ed.), Psychology: A study of a science, Vol. 4. New York: McGraw-Hill, 1962.

CURTIS, D. W. An empirical evaluation of a two-stage model for magnitude estimations. Paper read at Western Psychological Association Meeting, San Francisco, California, 1967.

CURTIS, D. W., ATTNEAVE, F., \& HARRINGTON, T. A test of a two-stage model of magnitude judgment. Perception \& Psychophysics, $1968,3(1 \mathrm{~A}), 25-31$.

EKMAN, G. Is the power law a special case of Fechner's Law? Perceptual \& Motor Skills, 1964, 19, 730 .

FAGOT, R. F. Alternative power laws for ratio scaling. Psychometrika, $1966,31,201-214$.

FAGOT, R. F., \& STEWART, M. R. An experimental comparison of stimulus and response translated power-functions for brightness. Perception \& Psychophysics, 1968, 3(4B), 297-305.

STEVENS, S. S. The psychophysics of sensory functioning. In W. S. Rosenblith (Ed.), Sensory communication: Contributions to a symposium on principles of sensory communication. MIT-Wiley, 196 I 
TORGERSON, W. S. Distances and ratios in psychophysical scaling. Acta Psychologica, 1961, 19, 201-205.

TREISMAN, M. Sensory scaling and the psychophysical law. Quarterly Journal of Psychology, 1964, 16, 11-22.

\section{NOTES}

1. This research was supported by NIH Grant MH 14116. Appreciation is expressed to John Wireman for his aid in the collecting and analyzing of the data.

2. Address: Department of Psychology, University of Alberta, Edmonton, Alberta, Canada.
3. In terms of the two stage model, translation on the response axis implies a function describing magnitude judgments of stimuli rather different from Fagot's (1966) Response Law, $J=a\left(\phi^{k}-t^{k}\right)$, where $t$ is the threshold. It would be instead the four-parameter equation, $\mathrm{J}=\mathbf{a}\left(\phi^{\mathrm{k}}-\mathrm{t}^{\mathrm{k}}\right)^{\mathrm{m}}$, a function that is not readily tested empirically.

4. An analysis of variance of $\log I$ as a function of $\log$ function values yields a significant F-ratio, reflecting the greater weight assigned to these small values when their logarithms are considered.

(Accepted for publication September 11, 1968.) 